\title{
CONGRUENCES MODULO POWERS OF 3 FOR 3- AND 9-COLORED GENERALIZED FROBENIUS PARTITIONS
}

\author{
LIUQUAN WANG
}

\begin{abstract}
Let $c \phi_{k}(n)$ be the number of $k$-colored generalized Frobenius partitions of $n$. We establish some infinite families of congruences for $c \phi_{3}(n)$ and $c \phi_{9}(n)$ modulo arbitrary powers of 3 , which refine the results of Kolitsch. For example, for $k \geq 3$ and $n \geq 0$, we prove that

$$
c \phi_{3}\left(3^{2 k} n+\frac{7 \cdot 3^{2 k}+1}{8}\right) \equiv 0 \quad\left(\bmod 3^{4 k+5}\right) .
$$

We give two different proofs to the congruences satisfied by $c \phi_{9}(n)$. One of the proofs uses an relation between $c \phi_{9}(n)$ and $c \phi_{3}(n)$ due to Kolitsch, for which we provide a new proof in this paper.
\end{abstract}

\section{INTRODUCTION}

In 1984, Andrews [1] introduced the concept of $k$-colored generalized Frobenius partitions. We first color all the nonnegative integers using "colors" denoted by $1,2, \cdots, k$. Then we impose an ordering on these colored integers as follows:

$$
0_{1} \prec 0_{2} \prec \cdots \prec 0_{k} \prec 1_{1} \prec 1_{2} \prec \cdots \prec 1_{k} \prec 2_{1} \prec 2_{2} \prec \cdots \prec 2_{k} \prec \cdots \text {. }
$$

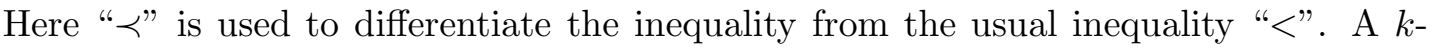
colored generalized Frobenius partition is a two-row array of colored integers of the form

$$
\left(\begin{array}{c}
a_{1}, a_{2}, \cdots, a_{r} \\
b_{1}, b_{2}, \cdots, b_{r}
\end{array}\right)
$$

such that

$$
a_{r} \prec a_{r-1} \prec \cdots \prec a_{1}, \quad b_{r} \prec b_{r-1} \prec \cdots \prec b_{1} .
$$

The number being partitioned by this partition is

$$
n=r+\sum_{i=1}^{r}\left(a_{i}+b_{i}\right) \text {. }
$$

For any positive integer $k$, Andrews [1] used the symbol $c \phi_{k}(n)$ to denote the number of $k$-colored generalized Frobenius partitions of $n$. Let

$$
\mathrm{C} \Phi_{k}(q):=\sum_{n=0}^{\infty} c \phi_{k}(n) q^{n} .
$$

Andrews [1] proved that

$$
\mathrm{C} \Phi_{k}(q)=\frac{1}{(q ; q)_{\infty}^{k}} \sum_{m_{1}, \cdots, m_{k-1}=-\infty}^{\infty} q^{Q\left(m_{1}, \cdots, m_{k-1}\right)},
$$

2010 Mathematics Subject Classification. Primary 05A17; Secondary 11P83.

Key words and phrases. Congruences; generalized Frobenius patitions; modulo powers of 3. 
where

$$
Q\left(m_{1}, \cdots, m_{k-1}\right)=\sum_{i=1}^{k-1} m_{i}^{2}+\sum_{1 \leq i<j \leq k-1} m_{i} m_{j}
$$

and

$$
(a ; q)_{\infty}=\prod_{n=0}^{\infty}\left(1-a q^{n}\right), \quad|q|<1 .
$$

To investigate arithmetic properties of $c \phi_{k}(n)$, Andrews [1] obtained alternative representations for $\mathrm{C}_{k}(q)$ with $k \in\{2,3,5\}$. In particular, for $k=3$, he [1, Eq. (9.4)] proved that

$$
\sum_{n=0}^{\infty} c \phi_{3}(n) q^{n}=\frac{1}{(q ; q)_{\infty}^{3}}\left(1+6 \sum_{n=0}^{\infty}\left(\frac{q^{3 n+1}}{1-q^{3 n+1}}-\frac{q^{3 n+2}}{1-q^{3 n+2}}\right)\right) .
$$

From the formulas of $\mathrm{C}_{k}(q)$, Andrews [1] found some nice properties of $c \phi_{k}(n)$. For instance, he proved that for $n \geq 0$,

$$
c \phi_{2}(5 n+3) \equiv 0 \quad(\bmod 5) .
$$

Since then, many congruences satisfied by $c \phi_{k}(n)$ have been discovered. Sellers 20] conjectured that (1.5) can be extended to a congruence modulo arbitrary powers of 5 . Namely, for any integers $k \geq 1$ and $n \geq 0$, he conjectured that

$$
c \phi_{2}\left(5^{k} n+\lambda_{k}\right) \equiv 0 \quad\left(\bmod 5^{k}\right),
$$

where $\lambda_{k}$ is the reciprocal of 12 modulo $5^{k}$. This conjecture was later proved by Paule and Radu [19] using the theory of modular forms.

After the work of Andrews, Kolitsch [12, 13] introduced the function $\overline{c \phi}_{k}(n)$, which denotes the number of $k$-colored generalized Frobenius partitions of $n$ whose order is $k$ under cyclic permutation of the colors. He [13] proved that for any positive integer $m$,

$$
\overline{c \phi}_{m}(n)=\sum_{d \mid(m, n)} \mu(d) c \phi_{\frac{m}{d}}\left(\frac{n}{d}\right)
$$

where $\mu(x)$ is the Möbius function. In particular, when $m$ is a prime, we have

$$
\overline{c \phi}_{m}(n)=c \phi_{m}(n)-p\left(\frac{n}{m}\right),
$$

where $p(n)$ is the ordinary partition function and we agree that $p(x)=0$ when $x$ is not an integer. Let $t_{k}$ be the reciprocal of 8 modulo $3^{k}$. Kolitsch [14] established the following infinite families of congruences: for $k \geq 1$ and $n \geq 0$,

$$
\overline{c \phi}_{3}\left(3^{k} n+t_{k}\right) \equiv 0 \begin{cases}\left(\bmod 3^{2 k+2}\right) & \text { if } k \text { is even } \\ \left(\bmod 3^{2 k+1}\right) & \text { if } k \text { is odd }\end{cases}
$$

From (1.8) we see that $c \phi_{3}(n)=\overline{c \phi}_{3}(n)$ if $n$ is not divisible by 3. Thus (1.9) implies that for $k \geq 1$ and $n \geq 0$,

$$
\begin{aligned}
c \phi_{3}\left(3^{2 k-1} n+\frac{5 \cdot 3^{2 k-1}+1}{8}\right) & \equiv 0 \quad\left(\bmod 3^{4 k-1}\right), \\
c \phi_{3}\left(3^{2 k} n+\frac{7 \cdot 3^{2 k}+1}{8}\right) & \equiv 0 \quad\left(\bmod 3^{4 k+2}\right) .
\end{aligned}
$$


In 1996, by using some combinatorial arguments, Kolitsch [15, Theorem 2] proved that for any $n \geq 1$,

$$
\overline{c \phi}_{9}(n)=3 \overline{c \phi}_{3}(3 n-1)
$$

Using (1.7), this relation is equivalent to

$$
c \phi_{9}(n)=3 c \phi_{3}(3 n-1)+c \phi_{3}\left(\frac{n}{3}\right) .
$$

From (1.10) and (1.11), Kolitsch [15, Corollary 3] used (1.12) to deduce the following congruences for $c \phi_{9}(n)$ : for $k \geq 1$ and $n \geq 0$,

$$
\begin{aligned}
c \phi_{9}\left(3^{2 k} n+\frac{5 \cdot 3^{2 k}+3}{8}\right) & \equiv 0 \quad\left(\bmod 3^{4 k-1}\right), \\
c \phi_{9}\left(3^{2 k+1} n+\frac{7 \cdot 3^{2 k+1}+3}{8}\right) & \equiv 0 \quad\left(\bmod 3^{4 k+2}\right) .
\end{aligned}
$$

In 2011 and 2015, Baruah and Sarmah [2, 3] found new representations of $\operatorname{C\Phi }_{k}(q)$ for $k \in\{4,5,6\}$. Moreover, they proved some congruences such as

$$
\begin{aligned}
& c \phi_{6}(3 n+1) \equiv 0 \quad(\bmod 9), \\
& c \phi_{6}(3 n+2) \equiv 0 \quad(\bmod 9) .
\end{aligned}
$$

In 2016, Gu, Wang and Xia [9] found many congruences modulo powers of 3 for $c \phi_{6}(n)$. For example, for any integer $n \geq 0$, we have that

$$
\begin{aligned}
c \phi_{6}(27 n+16) & \equiv 0 \quad\left(\bmod 3^{5}\right), \\
c \phi_{6}(243 n+142) & \equiv 0 \quad\left(\bmod 3^{6}\right) .
\end{aligned}
$$

Recently, Chan, Wang and Yang [6] used the theory of modular forms to give many new representations for $\mathrm{C}_{k}(q)$ for $k \leq 17$. In particular, a $q$-product representation for $\mathrm{C}_{9}(q)$ was discovered for the first time:

$$
\mathrm{C} \Phi_{9}(q)=\frac{E_{1}^{3}}{E_{3}^{4}}-240 q \frac{E_{9}^{3}}{E_{3}^{4}}+324 q \frac{E_{3}^{8}}{E_{1}^{9}}-1458 q^{2} \frac{E_{9}^{6}}{E_{1}^{3} E_{3}^{4}}+19683 q^{4} \frac{E_{9}^{12}}{E_{1}^{9} E_{3}^{4}},
$$

where we denote

$$
E_{k}=\left(q^{k} ; q^{k}\right)_{\infty}, \quad k \in \mathbb{N}
$$

for convenience. This representation leads to the congruences [6, Theorem 5.2]:

$$
\begin{aligned}
c \phi_{9}(9 n+3) \equiv 0 & (\bmod 9), \\
c \phi_{9}(9 n+6) \equiv 0 & (\bmod 9), \\
c \phi_{9}(3 n+1) \equiv 0 & (\bmod 81), \\
c \phi_{9}(3 n+2) \equiv 0 & (\bmod 729) .
\end{aligned}
$$

For more results on $k$-colored generalized Frobenius partitions, see [2, 3], [5]-[22], 24][26].

Following their steps, we are going to present more congruences modulo powers of 3 for $c \phi_{3}(n)$ and $c \phi_{9}(n)$. Observe that the cases $k=1$ and 2 of (1.10) and (1.11) give the following congruences:

$$
\begin{aligned}
& c \phi_{3}(3 n+2) \equiv 0 \quad\left(\bmod 3^{3}\right), \\
& c \phi_{3}(9 n+8) \equiv 0 \quad\left(\bmod 3^{6}\right), \\
& c \phi_{3}(27 n+17) \equiv 0 \quad\left(\bmod 3^{7}\right) \text {. }
\end{aligned}
$$


However, numerical evidences suggest that congruences (1.26) and (1.27) can be improved to

$$
\begin{aligned}
c \phi_{3}(9 n+8) & \equiv 0 & & \left(\bmod 3^{7}\right), \\
c \phi_{3}(27 n+17) & \equiv 0 & & \left(\bmod 3^{8}\right) .
\end{aligned}
$$

Moreover, there are some congruences which are not included in (1.10) and (1.11). For example, it appears that for any $n \geq 0$,

$$
\begin{aligned}
c \phi_{3}(9 n+5) & \equiv 0 & \left(\bmod 3^{5}\right), \\
c \phi_{3}(27 n+26) & \equiv 0 & \left(\bmod 3^{9}\right) .
\end{aligned}
$$

The above observations motivate us to improve and extend Kolitsch's congruences (1.10) and (1.11). First, we find some congruences modulo small powers of 3 .

Theorem 1.1. For any integer $n \geq 0$ we have

$$
\begin{aligned}
& c \phi_{3}(3 n+1) \equiv 0 \quad\left(\bmod 3^{2}\right), \\
& c \phi_{3}(3 n+2) \equiv 0 \quad\left(\bmod 3^{3}\right) \text {, } \\
& c \phi_{3}(9 n+5) \equiv 0 \quad\left(\bmod 3^{5}\right), \\
& c \phi_{3}(9 n+8) \equiv 0 \quad\left(\bmod 3^{7}\right), \\
& c \phi_{3}(27 n+17) \equiv 0 \quad\left(\bmod 3^{8}\right), \\
& c \phi_{3}(27 n+26) \equiv 0 \quad\left(\bmod 3^{9}\right) \text {, } \\
& c \phi_{3}(81 n+44) \equiv 0 \quad\left(\bmod 3^{10}\right), \\
& c \phi_{3}(81 n+71) \equiv 0 \quad\left(\bmod 3^{12}\right), \\
& c \phi_{3}(243 n+152) \equiv 0 \quad\left(\bmod 3^{13}\right) \text {, } \\
& c \phi_{3}(243 n+233) \equiv 0 \quad\left(\bmod 3^{14}\right) \text {. }
\end{aligned}
$$

All the moduli in congruences (1.32)-(1.41) cannot be replaced by higher powers of 3.

Then we give some general congruences beyond those in Theorem 1.1.

Theorem 1.2. For $k \geq 3$ and $n \geq 0$ we have

$$
\begin{array}{rlrl}
c \phi_{3}\left(3^{2 k} n+\frac{7 \cdot 3^{2 k}+1}{8}\right) & \equiv 0 & \left(\bmod 3^{4 k+5}\right), \\
c \phi_{3}\left(3^{2 k+1} n+\frac{5 \cdot 3^{2 k+1}+1}{8}\right) & \equiv 0 & \left(\bmod 3^{4 k+6}\right), \\
c \phi_{3}\left(3^{2 k+1} n+\frac{23 \cdot 3^{2 k}+1}{8}\right) & \equiv 0 & \left(\bmod 3^{4 k+7}\right), \\
c \phi_{3}\left(3^{2 k+2} n+\frac{13 \cdot 3^{2 k+1}+1}{8}\right) & \equiv 0 \quad\left(\bmod 3^{4 k+8}\right) .
\end{array}
$$

The second goal of this paper is to extend congruences (1.21) and (1.22) to larger families of congruences for $c \phi_{9}(n)$ and improve Kolitsch's congruences (1.14) and (1.15). We obtain results for $c \phi_{9}(n)$ which are similar to Theorems 1.1 and 1.2.

Theorem 1.3. For any integer $n \geq 0$ we have

$$
\begin{array}{ll}
c \phi_{9}(9 n+3) \equiv 0 & \left(\bmod 3^{2}\right), \\
c \phi_{9}(9 n+6) \equiv 0 & \left(\bmod 3^{3}\right),
\end{array}
$$




$$
\begin{array}{rlrl}
c \phi_{9}(27 n+15) & \equiv 0 & \left(\bmod 3^{5}\right), \\
c \phi_{9}(27 n+24) & \equiv 0 & \left(\bmod 3^{7}\right), \\
c \phi_{9}(81 n+51) & \equiv 0 & \left(\bmod 3^{8}\right), \\
c \phi_{9}(81 n+78) & \equiv 0 & \left(\bmod 3^{9}\right), \\
c \phi_{9}(243 n+132) & \equiv 0 & \left(\bmod 3^{10}\right), \\
c \phi_{9}(243 n+213) & \equiv 0 \quad\left(\bmod 3^{12}\right), \\
c \phi_{9}(729 n+456) & \equiv 0 \quad\left(\bmod 3^{13}\right), \\
c \phi_{9}(729 n+699) & \equiv 0 & \left(\bmod 3^{14}\right) .
\end{array}
$$

All the moduli in congruences (1.46) - (1.55) cannot be replaced by higher powers of 3.

Theorem 1.4. For $k \geq 3$ and $n \geq 0$ we have

$$
\begin{array}{rll}
c \phi_{9}\left(3^{2 k+1} n+\frac{7 \cdot 3^{2 k+1}+3}{8}\right) & \equiv 0 & \left(\bmod 3^{4 k+5}\right), \\
c \phi_{9}\left(3^{2 k+2} n+\frac{5 \cdot 3^{2 k+2}+3}{8}\right) & \equiv 0 & \left(\bmod 3^{4 k+6}\right), \\
c \phi_{9}\left(3^{2 k+2} n+\frac{23 \cdot 3^{2 k+1}+3}{8}\right) & \equiv 0 & \left(\bmod 3^{4 k+7}\right), \\
c \phi_{9}\left(3^{2 k+3} n+\frac{13 \cdot 3^{2 k+2}+3}{8}\right) & \equiv 0 & \left(\bmod 3^{4 k+8}\right) .
\end{array}
$$

The paper is organized as follows. In Section 2 we first collect some useful facts from the work of Kolitsch [14. Then we give proofs to Theorems 1.1 1.2, In Section 3 we present two different proofs to the congruences satisfied by $c \phi_{9}(n)$. The first proof uses (1.13) and Theorem 1.1 1.2, We will also give a new proof to (1.13) by comparing the generating functions of $c \phi_{3}(n)$ and $c \phi_{9}(n)$. In the second proof of Theorems 1.3 1.4, we do not use (1.13). Instead, we will establish congruences modulo powers of 3 for the coefficients in the series expansion of each term in (1.20).

\section{Congruences Modulo Powers of 3 For $c \phi_{3}(n)$}

Following the notation in [14], we define

$$
\xi=\frac{E_{1}^{3}}{q E_{9}^{3}}, \quad T=\frac{q^{3} E_{9}^{3}}{E_{3}^{3}}, \quad S=\frac{E_{3}^{4}}{E_{9}^{4}}+9 \frac{q^{3} E_{3} E_{27}^{3}}{E_{9}^{4}} .
$$

The functions $\xi$ and $T$ satisfy an modular equation of order 3 .

Lemma 2.1. (Cf. [14, Lemma 3]) We have

$$
\xi^{3}+9 \xi^{2}+27 \xi=q^{9} T^{-4},
$$

or equivalently,

$$
E_{1}^{9} E_{9}^{3}+9 q E_{1}^{6} E_{9}^{6}+27 q^{2} E_{1}^{3} E_{9}^{9}=E_{3}^{12} .
$$

Next we define an infinite matrix $\left(m_{i, j}\right)_{i, j \geq 1}$ by

(1) $m_{1,1}=3, m_{1, i}=0$ for $i \geq 2$;

(2) $m_{2,1}=1, m_{2,2}=3^{4}, m_{2, j}=0$ for $j \geq 3$;

(3) $m_{3,1}=0, m_{3,2}=2 \cdot 3^{3}, m_{3,3}=3^{7}, m_{3, j}=0$ for $j \geq 4$;

(4) $m_{i, j}=m_{i-3, j-1}+9 m_{i-2, j-1}+27 m_{i-1, j-1}$ for $i \geq 4, j \geq 2$. 
By induction on $i$, it is not difficult to show that

$$
m_{i, j}=0, \quad \text { if } \quad i \geq 3 j .
$$

This was first observed by Kolitsch [14, p. 346].

We define operators $H_{m, r}(0 \leq r \leq m-1)$ which act on infinite series as

$$
H_{m, r}\left(\sum_{n \in \mathbb{Z}} g(n) q^{n}\right):=\sum_{n \in \mathbb{Z}} g(m n+r) q^{m n+r} .
$$

The importance of $\left(m_{i, j}\right)_{i, j \geq 1}$ can be seen from the following lemma.

Lemma 2.2. (Cf. [14, Lemma 4]) We have

$$
H_{3,2}\left(\xi^{-i}\right)=\frac{S}{q} \sum_{j=1}^{\infty} m_{i, j} T^{4 j} q^{-9 j}
$$

This lemma can be proved by using Lemma 2.1. See [14, p. 346] for discussions.

Next we define two matrices $\left(a_{i, j}\right)_{i, j \geq 1}$ and $\left(b_{i, j}\right)_{i, j \geq 1}$ by

$$
\begin{aligned}
a_{i, j} & =9 m_{4 i+1, i+j}+m_{4 i, i+j}, \\
b_{i, j} & =m_{4 i-1, i+j}+9 m_{4 i, i+j} .
\end{aligned}
$$

From direct computations, we find that

$$
\begin{aligned}
& a_{1,1}=21, \quad a_{1,2}=10206, \quad a_{1,3}=767637, \quad a_{1,4}=14348907, \quad a_{1, j}=0, \quad j \geq 5, \\
& b_{1,1}=162, \quad b_{1,2}=21870, \quad b_{1,3}=531441, \quad b_{1, j}=0, \quad j \geq 4 .
\end{aligned}
$$

Lemma 2.3. (Cf. [14, Lemma 5].) We have

$$
\begin{aligned}
& H_{3,2}\left(9 \xi^{-4 i-1}+\xi^{-4 i}\right)=\frac{S}{q} \sum_{j=1}^{\infty} a_{i, j} T^{4 i+4 j} q^{-9 i-9 j} \\
& H_{3,2}\left(\xi^{-4 i+1}+9 \xi^{-4 i}\right)=\frac{S}{q} \sum_{j=1}^{\infty} b_{i, j} T^{4 i+4 j} q^{-9 i-9 j} .
\end{aligned}
$$

Now we define $\left(x_{i, j}\right)_{i, j \geq 1}$ by

$$
x_{1,1}=3, \quad x_{1, j}=0, \quad j \geq 2
$$

and for $k \geq 1$,

$$
\begin{aligned}
x_{2 k, j} & =\sum_{i=1}^{\infty} x_{2 k-1, i} b_{i, j}, \\
x_{2 k+1, j} & =\sum_{i=1}^{\infty} x_{2 k, i} a_{i, j} .
\end{aligned}
$$

In order to prove (1.9), Kolitsch [14, Theorem 2] established formulas for $\sum_{n=0}^{\infty} \overline{c \phi}_{3}\left(3^{k} n+\right.$ $\left.t_{k}\right) q^{n}$. In view of (1.8), his formulas can be restated as the following lemma.

Lemma 2.4. For $k \geq 1$, we have

$$
\sum_{n=0}^{\infty} c \phi_{3}\left(3^{2 k-1} n+\frac{5 \cdot 3^{2 k-1}+1}{8}\right) q^{n}=\frac{9 T}{q^{3} E_{3}} \sum_{j=1}^{\infty} x_{2 k-1, j} T^{-4 j} q^{9 j} \xi^{-4 j}(\xi+9)
$$


and

$$
\sum_{n=0}^{\infty} c \phi_{3}\left(3^{2 k} n+\frac{7 \cdot 3^{2 k}+1}{8}\right) q^{n}=\frac{9}{q E_{3}} \sum_{j=1}^{\infty} x_{2 k, j} T^{-4 j} q^{9 j} \xi^{-4 j-1}(\xi+9) .
$$

For any integer $n$, let $\pi(n)$ denote the 3 -adic order of $n$ and we agree that $\pi(0)=\infty$. For any real number $x$, we denote by $[x]$ the integer part of $x$. In order to establish the congruences (1.10) and (1.11), Kolitsch [14] examined the 3 -adic orders of $x_{i, j}$ for $i, j \geq 1$.

Lemma 2.5. (Cf. [14, Lemma 9].) We have

$$
\begin{aligned}
\pi\left(x_{1,1}\right) & =1, \\
\pi\left(x_{2 k, j}\right) & \geq 4 k+\left[\frac{9 j-9}{2}\right], \\
\pi\left(x_{2 k+1, j}\right) & \geq 4 k+1+\left[\frac{9 j-8}{2}\right] .
\end{aligned}
$$

It is then clear that when $j \geq 2$,

$$
\pi\left(x_{2 k, j}\right) \geq 4 k+4, \quad \pi\left(x_{2 k+1, j}\right) \geq 4 k+6 .
$$

To prove Theorems 1.1 and 1.2. we need to improve the estimate for $\pi\left(x_{k, 1}\right)$.

Lemma 2.6. For $k \geq 3$, we have

$$
\begin{aligned}
\pi\left(x_{2 k, 1}\right) & \geq 4 k+3 \\
\pi\left(x_{2 k+1,1}\right) & \geq 4 k+4 .
\end{aligned}
$$

Proof. By computations we find that

$$
a_{2,1}=1, \quad b_{2,1}=30, \quad b_{3,1}=1 .
$$

By (2.6) we have

$$
a_{i, 1}=9 m_{4 i+1, i+1}+m_{4 i, i+1} .
$$

By (2.3) we know that $a_{i, 1}=0$ if $i \geq 3$. Similarly by (2.7) we have

$$
b_{i, 1}=m_{4 i-1, i+1}+9 m_{4 i, i+1} .
$$

If $i \geq 4$, then by (2.3) we know $b_{i, 1}=0$.

By definition we have

$$
x_{2 k, 1}=162 x_{2 k-1,1}+30 x_{2 k-1,2}+x_{2 k-1,3}
$$

and

$$
x_{2 k+1,1}=21 x_{2 k, 1}+x_{2 k, 2} .
$$

Hence $x_{2,1}=2 \cdot 3^{5}$ and

$$
\pi\left(x_{3,1}\right) \geq \min \left\{1+\pi\left(x_{2,1}\right), \pi\left(x_{2,2}\right)\right\} .
$$

From (2.16) we see that $\pi\left(x_{2,2}\right) \geq 8$. Hence (2.23) implies $\pi\left(x_{3,1}\right) \geq 6$. Next, using Lemma 2.5 and (2.21) we see that

$$
\pi\left(x_{4,1}\right) \geq \min \left\{4+\pi\left(x_{3,1}\right), 1+\pi\left(x_{3,2}\right), \pi\left(x_{3,3}\right)\right\} \geq 10 .
$$

In the same way, we can prove that $\pi\left(x_{5,1}\right) \geq 11$ and $\pi\left(x_{6,1}\right) \geq 15$. Thus (2.17) is true for $k=3$. 
Suppose (2.17) is true for some $k \geq 3$. By (2.22) and (2.16) we deduce that

$$
\pi\left(x_{2 k+1,1}\right) \geq \min \left\{1+\pi\left(x_{2 k, 1}\right), \pi\left(x_{2 k, 2}\right)\right\} \geq 4 k+4 .
$$

Hence (2.18) is true for $k$. By (2.21) and Lemma 2.5 we deduce that

$$
\pi\left(x_{2 k+2,1}\right) \geq \min \left\{4+\pi\left(x_{2 k+1,1}\right), 1+\pi\left(x_{2 k+1,2}\right), \pi\left(x_{2 k+1,3}\right)\right\} \geq 4 k+7 .
$$

This implies that (2.17) is true for $k+1$. By induction on $k$ we complete the proof of Lemma 2.6.

Now we are able to prove Theorems 1.1 and 1.2 .

Proof of Theorem 1.1. From [12, Theorem 1] we have

$$
\overline{c \phi}_{3}(n) \equiv 0 \quad\left(\bmod 3^{2}\right) .
$$

Since $c \phi_{3}(n)=c \phi_{3}(n)$ when $n$ is not divisible by 3, congruence (1.32) follows.

Next, letting $k=1$ in (2.11), from (2.8) we deduce that

$$
c \phi_{3}(3 n+2) \equiv 0 \quad\left(\bmod 3^{3}\right) .
$$

By (2.11) and the binomial theorem, we have

$$
\begin{aligned}
\sum_{n=0}^{\infty} c \phi_{3}(3 n+2) q^{n} & =27\left(\frac{E_{3}^{8}}{E_{1}^{9}}+9 q \frac{E_{3}^{8} E_{9}^{3}}{E_{1}^{12}}\right) \\
& \equiv 27 E_{3}^{5} \quad\left(\bmod 3^{5}\right) .
\end{aligned}
$$

Since the terms of the form $q^{3 n+1}$ do not appear in the series expansion of $E_{3}^{5}$, we deduce that

$$
c \phi_{3}(9 n+5) \equiv 0 \quad\left(\bmod 3^{5}\right) .
$$

In the proof of Lemma 2.6 we have seen that $x_{2,1}=2 \cdot 3^{5}$. Moreover by (2.16) we know that $\pi\left(x_{2, j}\right) \geq 8$ for $j \geq 2$. Therefore by letting $k=1$ in (2.12) we deduce that

$$
\sum_{n=0}^{\infty} c \phi_{3}(9 n+8) q^{n} \equiv 2 \cdot 3^{7}\left(\frac{E_{3}^{11}}{E_{1}^{12}}+9 q \frac{E_{3}^{11} E_{9}^{3}}{E_{1}^{15}}\right) \quad\left(\bmod 3^{10}\right) .
$$

It follows that

$$
c \phi_{3}(9 n+8) \equiv 0 \quad\left(\bmod 3^{7}\right) .
$$

Moreover, by the binomial theorem, we deduce that

$$
\sum_{n=0}^{\infty} c \phi_{3}(9 n+8) q^{n} \equiv 2 \cdot 3^{7} \frac{E_{3}^{11}}{E_{1}^{12}} \equiv 2 \cdot 3^{7} \frac{E_{3}^{8}}{E_{1}^{3}} \quad\left(\bmod 3^{9}\right) .
$$

From [23, Lemma 2.6] we find

$$
\frac{1}{E_{1}^{3}}=\frac{E_{9}^{3}}{E_{3}^{12}}\left(E_{3}^{2} a^{2}\left(q^{3}\right)+3 q E_{3} a\left(q^{3}\right) E_{9}^{3}+9 q^{2} E_{9}^{6}\right)
$$

where

$$
a(q)=\left(1+6 \sum_{n=0}^{\infty}\left(\frac{q^{3 n+1}}{1-q^{3 n+1}}-\frac{q^{3 n+2}}{1-q^{3 n+2}}\right)\right) .
$$

Using (2.28), extracting the terms on both sides of (2.27) in which the exponent of $q$ is congruent to 2 modulo 3 , we deduce that

$$
c \phi_{3}(27 n+26) \equiv 0 \quad\left(\bmod 3^{9}\right) .
$$


Similarly, from (2.27) and (2.28) we deduce that

$$
c \phi_{3}(27 n+17) \equiv 0 \quad\left(\bmod 3^{8}\right) .
$$

In the proof of Lemma 2.6 we have seen that $\pi\left(x_{3,1}\right) \geq 6$. This together with (2.16) and (2.11) imply that

$$
\sum_{n=0}^{\infty} c \phi_{3}(27 n+17) q^{n} \equiv 9 x_{3,1}\left(\frac{E_{3}^{8}}{E_{1}^{9}}+9 q \frac{E_{3}^{8} E_{9}^{3}}{E_{1}^{12}}\right) \quad\left(\bmod 3^{12}\right) .
$$

By the binomial theorem, we deduce that

$$
\sum_{n=0}^{\infty} c \phi_{3}(27 n+17) q^{n} \equiv \frac{E_{3}^{8}}{E_{1}^{9}} \equiv 9 x_{3,1} E_{3}^{5} \quad\left(\bmod 3^{10}\right) .
$$

Hence by extracting the terms in which the exponent of $q$ is congruent to 1 modulo 3 , we obtain

$$
c \phi_{3}(81 n+44) \equiv 0 \quad\left(\bmod 3^{10}\right) .
$$

Let $k=2$ in (2.12). By (2.16), (2.24) and (2.28) we deduce that

$$
\begin{aligned}
\sum_{n=0}^{\infty} c \phi_{3}(81 n+71) q^{n} & \equiv 9 x_{4,1}\left(\frac{E_{3}^{11}}{E_{1}^{12}}+9 q \frac{E_{3}^{11} E_{9}^{3}}{E_{1}^{15}}\right) \\
& \equiv 9 x_{4,1} \frac{E_{3}^{11}}{E_{1}^{9}} \cdot \frac{1}{E_{1}^{3}} \\
& \equiv 9 x_{4,1} \frac{E_{9}^{3}}{E_{3}^{4}}\left(E_{3}^{2} a^{2}\left(q^{3}\right)+3 q E_{3} a\left(q^{3}\right) E_{9}^{3}+9 q^{2} E_{9}^{6}\right) \quad\left(\bmod 3^{14}\right) .
\end{aligned}
$$

It follows that

$$
c \phi_{3}(81 n+71) \equiv 0 \quad\left(\bmod 3^{12}\right)
$$

Moreover, extracting the terms in which the exponent of $q$ is congruent to 1 and 2 on both sides of (2.32), we obtain (1.40) and (1.41), respectively.

Proof of Theorem 1.2. By Lemma 2.5 we have $\pi\left(x_{2 k, 2}\right) \geq 4 k+4$ and

$$
\pi\left(x_{2 k, j}\right) \geq 4 k+9, \quad j \geq 3 .
$$

By (2.12) and (2.16) we deduce that

$$
\begin{aligned}
& \sum_{n=0}^{\infty} c \phi_{3}\left(3^{2 k} n+\frac{7 \cdot 3^{2 k}+1}{8}\right) q^{n} \\
\equiv & 9 x_{2 k, 1}\left(\frac{E_{3}^{11}}{E_{1}^{12}}+9 q \frac{E_{3}^{11} E_{9}^{3}}{E_{1}^{15}}\right)+9 x_{2 k, 2} q \frac{E_{3}^{23}}{E_{1}^{24}}\left(\bmod 3^{4 k+8}\right) .
\end{aligned}
$$

For $k \geq 3$, by Lemma 2.6 we have $\pi\left(x_{2 k, 1}\right) \geq 4 k+3$ and hence

$$
c \phi_{3}\left(3^{2 k} n+\frac{7 \cdot 3^{2 k}+1}{8}\right) \equiv 0 \quad\left(\bmod 3^{4 k+5}\right) .
$$

Moreover, by (2.33) and the binomial theorem, we deduce that

$$
\sum_{n=0}^{\infty} c \phi_{3}\left(3^{2 k} n+\frac{7 \cdot 3^{2 k}+1}{8}\right) q^{n} \equiv 9 x_{2 k, 1} E_{3}^{7} \quad\left(\bmod 3^{4 k+6}\right) .
$$


It follows that

$$
c \phi_{3}\left(3^{2 k}(3 n+1)+\frac{7 \cdot 3^{2 k}+1}{8}\right) \equiv 0 \quad\left(\bmod 3^{4 k+6}\right),
$$

which proves (1.43).

By (2.33) and the binomial theorem again, we have

$$
\sum_{n=0}^{\infty} c \phi_{3}\left(3^{2 k} n+\frac{7 \cdot 3^{2 k}+1}{8}\right) q^{n} \equiv 9 x_{2 k, 1} \frac{E_{3}^{8}}{E_{1}^{3}}+9 x_{2 k, 2} q E_{3}^{15} \quad\left(\bmod 3^{4 k+7}\right) .
$$

Now recalling (2.28) and extracting the terms on both sides of (2.34) in which the exponent of $q$ is congruent to 2 modulo 3, we obtain (1.44).

By (2.16), (2.18) and (2.11) we deduce that

$$
\sum_{n=0}^{\infty} c \phi_{3}\left(3^{2 k+1} n+\frac{5 \cdot 3^{2 k+1}+1}{8}\right) q^{n} \equiv 9 x_{2 k+1,1} \frac{E_{3}^{8}}{E_{1}^{9}} \equiv 9 x_{2 k+1,1} E_{3}^{5} \quad\left(\bmod 3^{4 k+8}\right) .
$$

Extracting the terms on both sides of (2.35) in which the exponent of $q$ is congruent to 1 modulo 3 , we obtain (1.45).

\section{Congruences Modulo Powers of 3 For $c \phi_{9}(n)$}

In this section, we present two different proofs for Theorems 1.3 and 1.4

Our first proof is based on Kolitsch's relation (1.13). Kolitsch's original proof of (1.13) uses combinatorial arguments. Here we will give a new proof by series manipulations.

Lemma 3.1. We have

$$
a(q)=\frac{E_{1}^{3}}{E_{3}}+9 q \frac{E_{9}^{3}}{E_{3}}
$$

and

$$
a^{3}(q)=\frac{E_{1}^{9}}{E_{3}^{3}}+27 q \frac{E_{3}^{9}}{E_{1}^{3}} .
$$

Proof. It was proved in [4, Eqs. (2.3), (2.4), (2.11)] that

$$
\begin{aligned}
& a(q)=3 a\left(q^{3}\right)-2 \frac{E_{1}^{3}}{E_{3}}, \\
& a(q)=a\left(q^{3}\right)+6 q \frac{E_{9}^{3}}{E_{3}} .
\end{aligned}
$$

By eliminating $a\left(q^{3}\right)$ from (3.3) and (3.4), we obtain (3.1).

The identity (3.2) follows from [4, Proposition 2.2, Theorem 2.3]. It also follows by taking the cubic power on both sides of (3.1) and then using (2.2) to do simplifications.

Proof of (1.13). It suffices to show that

$$
\sum_{n=0}^{\infty} c \phi_{9}(n) q^{n}=\sum_{n=0}^{\infty} c \phi_{3}\left(\frac{n}{3}\right) q^{n}+3 \sum_{n=0}^{\infty} c \phi_{3}(3 n-1) q^{n} .
$$

Using (3.1) and (1.4) we obtain

$$
\sum_{n=0}^{\infty} c \phi_{3}(n) q^{n}=\frac{1}{E_{3}}+9 q \frac{E_{9}^{3}}{E_{1}^{3} E_{3}}
$$


Combining this identity with (2.25) we deduce that

$$
\sum_{n=0}^{\infty} c \phi_{3}\left(\frac{n}{3}\right) q^{n}+3 \sum_{n=0}^{\infty} c \phi_{3}(3 n-1) q^{n}=\frac{1}{E_{9}}+9 q^{3} \frac{E_{27}^{3}}{E_{3}^{3} E_{9}}+81 q \frac{E_{3}^{8}}{E_{1}^{9}}+3^{6} q^{2} \frac{E_{3}^{8} E_{9}^{3}}{E_{1}^{12}} .
$$

Multiplying by $E_{1}^{9}$ on both sides of (1.20) and (3.7), we know that (3.5) is equivalent to

$$
F_{1}(q)=F_{2}(q)
$$

where

$$
\begin{aligned}
& F_{1}(q)=\frac{E_{1}^{12}}{E_{3}^{4}}-240 q \frac{E_{1}^{9} E_{9}^{3}}{E_{3}^{4}}+243 q E_{3}^{8}-1458 q^{2} \frac{E_{1}^{6} E_{9}^{6}}{E_{3}^{4}}+19683 q^{4} \frac{E_{9}^{12}}{E_{3}^{4}}, \\
& F_{2}(q)=\frac{E_{1}^{9}}{E_{9}}+9 q^{3} \frac{E_{1}^{9} E_{27}^{3}}{E_{3}^{3} E_{9}}+3^{6} q^{2} \frac{E_{3}^{8} E_{9}^{3}}{E_{1}^{3}} .
\end{aligned}
$$

By [23, Lemma 2.5] we have

$$
E_{1}^{3}=E_{3} a\left(q^{3}\right)-3 q E_{9}^{3} .
$$

Substituting (2.28) and (3.11) into (3.9), after simplification, we obtain

$$
\begin{aligned}
& H_{3,0}\left(F_{1}(q)\right)=a^{4}\left(q^{3}\right)+2160 q^{3} \frac{E_{9}^{9}}{E_{3}^{3}} a\left(q^{3}\right), \\
& H_{3,1}\left(F_{1}(q)\right)=243 q E_{3}^{8}-252 q \frac{E_{9}^{3}}{E_{3}} a^{3}\left(q^{3}\right)+13122 q^{4} \frac{E_{9}^{12}}{E_{3}^{4}}, \\
& H_{3,2}\left(F_{1}(q)\right)=756 q^{2} \frac{E_{9}^{6}}{E_{3}^{2}} a^{2}\left(q^{3}\right) .
\end{aligned}
$$

Similarly, substituting (2.28) and (3.11) into (3.10), after simplification, we obtain

$$
\begin{aligned}
& H_{3,0}\left(F_{2}(q)\right)=\frac{E_{3}^{3}}{E_{9}} a^{3}\left(q^{3}\right)+9 q^{3} \frac{E_{27}^{3}}{E_{9}} a^{3}\left(q^{3}\right)-27 q^{3} E_{9}^{8}+2187 q^{3} \frac{E_{9}^{9}}{E_{3}^{3}} a\left(q^{3}\right)-243 q^{6} \frac{E_{9}^{8} E_{27}^{3}}{E_{3}^{3}}, \\
& H_{3,1}\left(F_{2}(q)\right)=-9 q E_{3}^{2} E_{9}^{2} a^{2}\left(q^{3}\right)-81 q^{4} \frac{E_{9}^{2} E_{27}^{3}}{E_{3}} a^{2}\left(q^{3}\right)+6561 q^{4} \frac{E_{9}^{12}}{E_{3}^{4}}, \\
& H_{3,2}\left(F_{2}(q)\right)=27 q^{2} E_{3} E_{9}^{5} a\left(q^{3}\right)+729 q^{2} \frac{E_{9}^{6}}{E_{3}^{2}} a^{2}\left(q^{3}\right)+243 q^{5} \frac{E_{9}^{5} E_{27}^{3}}{E_{3}^{2}} a\left(q^{3}\right) .
\end{aligned}
$$

Comparing (3.12) with (3.15), we see that $H_{3,0}\left(F_{1}(q)\right)=H_{3,0}\left(F_{2}(q)\right)$ is equivalent to

$$
\left(a^{3}\left(q^{3}\right)-27 q^{3} \frac{E_{9}^{9}}{E_{3}^{3}}\right)\left(a\left(q^{3}\right)-\frac{E_{3}^{3}}{E_{9}}-9 q^{3} \frac{E_{27}^{3}}{E_{9}}\right)=0,
$$

which follows from (3.1).

Comparing (3.13) with (3.16) and replacing $q^{3}$ by $q$, after simplification, we see that $H_{3,1}\left(F_{1}(q)\right)=H_{3,1}\left(F_{2}(q)\right)$ is equivalent to

$$
-28 E_{3}^{3} a^{3}(q)+27 E_{1}^{9}+729 q \frac{E_{3}^{12}}{E_{1}^{3}}+9 q E_{3}^{2} E_{9}^{3} a^{2}(q)+E_{1}^{3} E_{3}^{2} a^{2}(q)=0 .
$$

Using (3.1), we observe that

$$
9 q E_{3}^{2} E_{9}^{3} a^{2}(q)+E_{1}^{3} E_{3}^{2} a^{2}(q)=E_{3}^{3} a^{2}(q)\left(\frac{E_{1}^{3}}{E_{3}}+9 q \frac{E_{9}^{3}}{E_{3}}\right)=E_{3}^{3} a^{3}(q) .
$$


Therefore, (3.19) is the same as

$$
-E_{3}^{3} a^{3}(q)+E_{1}^{9}+27 q \frac{E_{3}^{12}}{E_{1}^{3}}=0,
$$

which follows from (3.2). Hence $H_{3,1}\left(F_{1}(q)\right)=H_{3,1}\left(F_{2}(q)\right)$.

Next, comparing (3.14) with (3.17), we know that $H_{3,2}\left(F_{1}(q)\right)=H_{3,2}\left(F_{2}(q)\right)$ is equivalent to

$$
E_{3} a(q)=E_{1}^{3}+9 q E_{9}^{3}
$$

which follows from (3.1).

Thus we have proved that $H_{3, r}\left(F_{1}(q)\right)=H_{3, r}\left(F_{2}(q)\right)$ for $r \in\{0,1,2\}$. Hence (3.8) holds and our proof is complete.

Remark 1. The relation (1.13) can also be proved using the theory of modular forms. Let $q=e^{2 \pi i \tau}$ with $\operatorname{Im} \tau>0$. We denote by $M_{k}\left(\Gamma_{0}(N)\right)$ the space of modular forms with weight $k$ on $\Gamma_{0}(N)$. It is not difficult to show that both $F_{1}(q)$ and $F_{2}(q)$ are in $M_{4}\left(\Gamma_{0}(27)\right)$. Since $\operatorname{dim} M_{4}\left(\Gamma_{0}(27)\right)=12$. By checking that the first 12 coefficients of $F_{1}(q)$ and $F_{2}(q)$ agree with each other, we immediately prove that $F_{1}(q)=F_{2}(q)$.

Now we are able to prove Theorems 1.3 and 1.4 .

First Proof of Theorems 1.3 and 1.4. From (1.13) we have

$$
c \phi_{9}(3 n)=3 c \phi_{3}(9 n-1)+c \phi_{3}(n) .
$$

It is then clear that all the congruences in Theorems 1.3 and 1.4 follow from the congruences in Theorems 1.1 and 1.2. For example, (3.23) implies

$$
\begin{aligned}
& c \phi_{9}\left(3^{2 k+1} n+\frac{7 \cdot 3^{2 k+1}+3}{8}\right) \\
= & 3 c \phi_{3}\left(3^{2 k+2} n+\frac{7 \cdot 3^{2 k+2}+1}{8}\right)+c \phi_{3}\left(3^{2 k} n+\frac{7 \cdot 3^{2 k}+1}{8}\right) .
\end{aligned}
$$

This together with (1.42) implies (1.56). Other congruences can be proved in a similar fashion.

We can also give another proof without using Kolitsch's relation (1.13). For this we examine the terms in (1.20) one by one. We define five sequences $a_{i}(n)(1 \leq i \leq 5)$ by

$$
\begin{aligned}
& \sum_{n=0}^{\infty} a_{1}(n) q^{n}=\frac{E_{1}^{3}}{E_{3}^{4}}, \\
& \sum_{n=0}^{\infty} a_{2}(n) q^{n}=q \frac{E_{9}^{3}}{E_{3}^{4}}, \\
& \sum_{n=0}^{\infty} a_{3}(n) q^{n}=q \frac{E_{3}^{8}}{E_{1}^{9}}, \\
& \sum_{n=0}^{\infty} a_{4}(n) q^{n}=q^{2} \frac{E_{9}^{6}}{E_{1}^{3} E_{3}^{4}}, \\
& \sum_{n=0}^{\infty} a_{5}(n) q^{n}=q^{4} \frac{E_{9}^{12}}{E_{1}^{9} E_{3}^{4}} .
\end{aligned}
$$


Then (1.20) implies

$$
c \phi_{9}(n)=a_{1}(n)-240 a_{2}(n)+324 a_{3}(n)-1458 a_{4}(n)+19683 a_{5}(n) .
$$

By (3.11) we have

$$
\sum_{n=0}^{\infty} a_{1}(3 n) q^{n}=\frac{a(q)}{E_{1}^{3}}
$$

Comparing this with (1.4), we find that

$$
a_{1}(3 n)=c \phi_{3}(n) .
$$

According to the definition, we have $a_{2}(3 n)=0$. Hence

$$
c \phi_{9}(3 n)=c \phi_{3}(n)+324 a_{3}(3 n)-1458 a_{4}(3 n)+19683 a_{5}(3 n) .
$$

Now we establish some results which are analogous to Lemma 2.4.

Lemma 3.2. For $k \geq 1$ we have

(1)

$$
\sum_{n=0}^{\infty} a_{3}\left(3^{2 k} n+\frac{5 \cdot 3^{2 k}+3}{8}\right) q^{n}=9 \frac{T}{q^{3} E_{3}} \sum_{j=1}^{\infty} y_{2 k-1, j} T^{-4 j} q^{9 j} \xi^{-4 j}(\xi+9)
$$

and

$$
\sum_{n=0}^{\infty} a_{3}\left(3^{2 k+1} n+\frac{7 \cdot 3^{2 k+1}+3}{8}\right) q^{n}=9 \frac{1}{q E_{3}} \sum_{j=1}^{\infty} y_{2 k, j} T^{-4 j} q^{9 j} \xi^{-4 j-1}(\xi+9),
$$

where

$$
y_{1, j}=6 a_{1, j}+243 a_{2, j}, \quad j \geq 1
$$

and for $k \geq 1$,

$$
y_{2 k, j}=\sum_{i=1}^{\infty} y_{2 k-1, i} b_{i, j}, \quad y_{2 k+1, j}=\sum_{i=1}^{\infty} y_{2 k, i} a_{i, j}
$$

(2)

$$
\sum_{n=0}^{\infty} a_{4}\left(3^{2 k} n+\frac{5 \cdot 3^{2 k}+3}{8}\right) q^{n}=3 q^{3} \frac{E_{9}^{15}}{E_{3}^{16}} \sum_{j=1}^{\infty} z_{2 k-1, j} T^{-4 j-4} q^{9 j+9} \xi^{-4 j}(\xi+9)
$$

and for $k \geq 1$,

$$
\sum_{n=0}^{\infty} a_{4}\left(3^{2 k+1} n+\frac{7 \cdot 3^{2 k+1}+3}{8}\right) q^{n}=3 \frac{E_{3}^{11}}{q^{4} E_{9}^{12}} \sum_{j=1}^{\infty} z_{2 k, j} T^{4-4 j} q^{9 j-9} \xi^{-4 j-1}(\xi+9),
$$

where

$$
z_{1,1}=21, \quad z_{1,2}=2 \cdot 3^{6} \cdot 7, \quad z_{1,3}=3^{10} \cdot 13, \quad z_{1,4}=3^{15}, \quad z_{1, j}=0, \quad j \geq 5
$$

and for $k \geq 1$,

$$
z_{2 k, j}=\sum_{i=1}^{\infty} z_{2 k-1, i} a_{i, j}, \quad z_{2 k+1, j}=\sum_{i=1}^{\infty} z_{2 k, i} b_{i, j} .
$$


(3)

$$
\sum_{n=0}^{\infty} a_{5}\left(3^{2 k} n+\frac{5 \cdot 3^{2 k}+3}{8}\right) q^{n}=9 q^{3} \frac{E_{9}^{15}}{E_{3}^{16}} \sum_{j=1}^{\infty} w_{2 k-1, j} T^{-4 j-4} q^{9 j+9} \xi^{-4 j}(\xi+9)
$$

and

$$
\sum_{n=0}^{\infty} a_{5}\left(3^{2 k+1} n+\frac{7 \cdot 3^{2 k+1}+3}{8}\right) q^{n}=9 \frac{E_{3}^{11}}{q^{4} E_{9}^{12}} \sum_{j=1}^{\infty} w_{2 k, j} T^{4-4 j} q^{9 j-9} \xi^{-4 j-1}(\xi+9),
$$

where

$$
w_{1, j}=6 a_{2, j}+243 a_{3, j}, \quad j \geq 1
$$

and

$$
w_{2 k, j}=\sum_{i=1}^{\infty} w_{2 k-1, i} a_{i, j}, \quad w_{2 k+1, j}=\sum_{i=1}^{\infty} w_{2 k, i} b_{i, j} .
$$

Proof. The proofs of (1)-(3) are similar to the proof of Lemma 2.4 given in [14]. Here we only give the details for (1).

We proceed by induction on $k$. We write

$$
\sum_{n=0}^{\infty} a_{3}(n) q^{n+2}=\frac{E_{3}^{8}}{E_{9}^{9}} \xi^{-3}
$$

Applying the operator $H_{3,2}$ to both sides, we obtain

$$
\sum_{n=0}^{\infty} a_{3}(3 n) q^{3 n}=\frac{E_{3}^{8} S}{q^{3} E_{9}^{9}}\left(54 T^{8} q^{-18}+2187 T^{12} q^{-27}\right) .
$$

Replacing $q^{3}$ by $q$, using the fact that

$$
T\left(q^{1 / 3}\right)=\frac{q E_{3}^{3}}{E_{1}^{3}}=T^{-1} \xi^{-1} q^{3}, \quad S\left(q^{1 / 3}\right)=\frac{E_{1}^{4}}{E_{3}^{4}}\left(1+9 \xi^{-1}\right),
$$

we obtain

$$
\sum_{n=0}^{\infty} a_{3}(3 n) q^{n}=\frac{9}{E_{3}}\left(6 T^{-4} q^{9} \xi^{-5}+243 T^{-8} q^{18} \xi^{-9}\right)(\xi+9) .
$$

Applying $H_{3,2}$ to both sides of (3.45), we get

$$
\begin{aligned}
\left.\sum_{n=0}^{\infty} a_{3}(9 n+6)\right) q^{3 n+2} & =\frac{9}{E_{3}}\left(6 T^{-4} q^{9} H_{3,2}\left(9 \xi^{-5}+\xi^{-4}\right)+243 T^{-8} q^{18} H_{3,2}\left(9 \xi^{-9}+\xi^{-8}\right)\right) \\
& =\frac{9 S}{q E_{3}}\left(6 T^{-4} q^{9} \sum_{j=1}^{\infty} a_{1, j} T^{4+4 j} q^{-9-9 j}+243 T^{-8} q^{18} \sum_{j=1}^{\infty} a_{2, j} T^{8+4 j} q^{-18-9 j}\right) \\
& =\frac{9 S}{q E_{3}} \sum_{j=1}^{\infty} y_{1, j} T^{4 j} q^{-9 j}
\end{aligned}
$$

Now dividing both sides by $q^{2}$ and replacing $q^{3}$ by $q$, using (3.44) we obtain

$$
\sum_{n=0}^{\infty} a_{3}(9 n+6) q^{n}=\frac{9 T}{q^{3} E_{3}} \sum_{j=1}^{\infty} y_{1, j} T^{-4 j} q^{9 j} \xi^{-4 j}(\xi+9) .
$$

This proves (3.34) for $k=1$. 
Suppose (3.34) is true for some integer $k \geq 1$. Extracting the terms in which the exponent of $q$ is congruent to 2 modulo 3 , we obtain

$\sum_{n=0}^{\infty} a_{3}\left(3^{2 k}(3 n+2)+\frac{5 \cdot 3^{2 k}+3}{8}\right) q^{3 n+2}=\frac{9 T}{q^{3} E_{3}} \sum_{i=1}^{\infty} y_{2 k-1, i} T^{-4 i} q^{9 i} H_{3,2}\left(\xi^{-4 i+1}+9 \xi^{-4 i}\right)$.

Using Lemma 2.3 it follows that

$$
\sum_{n=0}^{\infty} a_{3}\left(3^{2 k+1} n+\frac{7 \cdot 3^{2 k+1}+3}{8}\right) q^{3 n+2}=\frac{9 T S}{q^{4} E_{3}} \sum_{j=1}^{\infty} y_{2 k, j} T^{4 j} q^{-9 j} .
$$

Dividing both sides by $q^{2}$ and then replacing $q^{3}$ by $q$, using (3.44) we obtain (3.35).

Next, extracting the terms in which the exponent of $q$ is congruent to 1 modulo 3 on both sides of (3.35), we have

$$
\begin{aligned}
& \sum_{n=0}^{\infty} c \phi_{3}\left(3^{2 k+1}(3 n+1)+\frac{7 \cdot 3^{2 k+1}+3}{8}\right) q^{3 n+1} \\
& =\frac{9}{q E_{3}} \sum_{i=1}^{\infty} y_{2 k, i} T^{-4 i} q^{9 i} H_{3,2}\left(9 \xi^{-4 i-1}+\xi^{-4 i}\right) .
\end{aligned}
$$

Using Lemma 2.3 it follows that

$$
\sum_{n=0}^{\infty} c \phi_{3}\left(3^{2 k+2} n+\frac{5 \cdot 3^{2 k+2}+3}{8}\right) q^{3 n+1}=\frac{9 S}{q^{2} E_{3}} \sum_{j=1}^{\infty} y_{2 k+1, j} T^{4 j} q^{-9 j}
$$

Dividing both sides by $q$, then replacing $q^{3}$ by $q$ and using (3.44), we obtain

$$
\sum_{n=0}^{\infty} c \phi_{3}\left(3^{2 k+2} n+\frac{5 \cdot 3^{2 k+2}+3}{8}\right) q^{n}=\frac{9 T}{q^{3} E_{3}} \sum_{j=1}^{\infty} y_{2 k+1, j} T^{-4 j} q^{9 j} \xi^{-4 j}(\xi+9) .
$$

This gives (3.34) with $k$ replaced by $k+1$. By induction on $k$ we complete the proof of (1).

In order to establish congruences modulo arbitrary powers of 3 for $c \phi_{9}(n)$, we need to examine the 3 -adic orders of $y_{i, j}, z_{i, j}$ and $w_{i, j}$. The following information about the 3 -adics orders of $a_{i, j}$ and $b_{i, j}$ will be helpful.

Lemma 3.3. (Cf. [14, Lemma 8].) We have

$$
\pi\left(a_{i, j}\right) \geq\left[\frac{9 j-3 i-3}{2}\right], \quad \pi\left(b_{i, j}\right) \geq\left[\frac{9 j-3 i}{2}\right] .
$$

Lemma 3.4. For any integer $k \geq 1$ we have

(1)

$$
\pi\left(y_{2 k-1, j}\right) \geq 4 k-2+\left[\frac{9 j-8}{2}\right], \quad \pi\left(y_{2 k, j}\right) \geq 4 k+1+\left[\frac{9 j-9}{2}\right]
$$

$$
\pi\left(z_{2 k-1, j}\right) \geq 4 k-3+\left[\frac{9 j-8}{2}\right], \quad \pi\left(z_{2 k, j}\right) \geq 4 k+\left[\frac{9 j-9}{2}\right]
$$


$(3)$

$$
\pi\left(w_{2 k-1, j}\right) \geq 4 k-3+\left[\frac{9 j-8}{2}\right], \quad \pi\left(w_{2 k, j}\right) \geq 4 k+\left[\frac{9 j-9}{2}\right] .
$$

Proof. We only prove (1) in details. The proofs of (2) and (3) are similar.

To prove (1) we use induction on $k$. For $k=1$, by (3.36) and Lemma 3.3 we find that

$$
\pi\left(y_{1, j}\right) \geq \min \left\{1+\left[\frac{9 j-6}{2}\right], 5+\left[\frac{9 j-9}{2}\right]\right\}=2+\left[\frac{9 j-8}{2}\right] .
$$

Hence the first inequality in (1) is true for $k=1$.

Suppose the first inequality in (1) is true for some $k \geq 1$. From (3.37) we have

$$
\pi\left(y_{2 k, j}\right) \geq \min _{i \geq 1} \pi\left(y_{2 k-1, i} b_{i, j}\right) \geq \min _{i \geq 1}\left(4 k-2+\left[\frac{9 i-8}{2}\right]+\left[\frac{9 j-3 i}{2}\right]\right) .
$$

Let

$$
f(i, j)=\left[\frac{9 i-8}{2}\right]+\left[\frac{9 j-3 i}{2}\right] .
$$

If we increase $i$ by 1 , the value of $\left[\frac{9 i-8}{2}\right]$ increases by at least 4 and the value of $\left[\frac{9 j-3 i}{2}\right]$ decreases by at most 2 . Thus $f(i, j)$ is increasing with respect to $i$. So the minimal value of $f(i, j)$ occurs when $i=1$. Therefore, from (3.54) we obtain

$$
\pi\left(y_{2 k, j}\right) \geq 4 k-2+\left[\frac{9 j-3}{2}\right]=4 k+1+\left[\frac{9 j-9}{2}\right] .
$$

Hence the second inequality in (1) is true for $k$.

Similarly, we have

$$
\begin{aligned}
\pi\left(y_{2 k+1, j}\right) & \geq \min _{i \geq 1}\left(\pi\left(y_{2 k, i}\right)+\pi\left(a_{i, j}\right)\right) \\
& \geq \min _{i \geq 1} 4 k+1+\left[\frac{9 i-9}{2}\right]+\left[\frac{9 j-3 i-3}{2}\right] \\
& \geq 4 k+2+\left[\frac{9 j-8}{2}\right] .
\end{aligned}
$$

This proves the first inequality of (1) for $k+1$. By induction we know (1) is true for any $k \geq 1$.

Lemma 3.5. For any integers $k \geq 1$ and $n \geq 0$ we have

$$
\begin{aligned}
a_{3}\left(3^{2 k} n+\frac{5 \cdot 3^{2 k}+3}{8}\right) & \equiv 0 \quad\left(\bmod 3^{4 k}\right), \\
a_{3}\left(3^{2 k+1} n+\frac{7 \cdot 3^{2 k+1}+3}{8}\right) & \equiv 0 \quad\left(\bmod 3^{4 k+3}\right) . \\
a_{4}\left(3^{2 k} n+\frac{5 \cdot 3^{2 k}+3}{8}\right) & \equiv 0 \quad\left(\bmod 3^{4 k-2}\right), \\
a_{4}\left(3^{2 k+1} n+\frac{7 \cdot 3^{2 k+1}+3}{8}\right) & \equiv 0 \quad\left(\bmod 3^{4 k+1}\right) . \\
a_{5}\left(3^{2 k} n+\frac{5 \cdot 3^{2 k}+3}{8}\right) & \equiv 0 \quad\left(\bmod 3^{4 k-1}\right), \\
a_{5}\left(3^{2 k+1} n+\frac{7 \cdot 3^{2 k+1}+3}{8}\right) & \equiv 0 \quad\left(\bmod 3^{4 k+2}\right) .
\end{aligned}
$$


Proof. The assertions follow directly from Lemmas 3.2 and 3.4 .

Proofs of Theorems 1.3 and 1.4. Recall from (3.33) that

$$
c \phi_{9}(3 n)=c \phi_{3}(n)+4 \cdot 3^{4} a_{3}(3 n)-2 \cdot 3^{6} a_{4}(3 n)+3^{9} a_{5}(3 n) .
$$

Congruences (1.46) and (1.47) follow from (1.32) and (1.33), respectively.

From (3.56), (3.58), (3.60) and (3.61) we deduce that for any $k \geq 1$,

$$
c \phi_{9}\left(3\left(3^{2 k} n+\frac{7 \cdot 3^{2 k}+1}{8}\right)\right) \equiv c \phi_{3}\left(3^{2 k} n+\frac{7 \cdot 3^{2 k}+1}{8}\right) \quad\left(\bmod 3^{4 k+7}\right) .
$$

Similarly, from (3.55), (3.57), (3.59) and (3.61) we deduce that for any $k \geq 0$,

$$
c \phi_{9}\left(3\left(3^{2 k+1} n+\frac{5 \cdot 3^{2 k+1}+1}{8}\right)\right) \equiv c \phi_{3}\left(3^{2 k+1} n+\frac{5 \cdot 3^{2 k+1}+1}{8}\right) \quad\left(\bmod 3^{4 k+8}\right) .
$$

Theorem 1.3 then follows from (3.62), (3.63) and Theorem 1.1. For example, let $k=0$ in (3.63). We have

$$
c \phi_{9}(9 n+6) \equiv c \phi_{3}(3 n+2) \quad\left(\bmod 3^{8}\right) .
$$

Replacing $n$ by $3 n+1$ and $3 n+2$ in (3.64), we obtain (1.48) and (1.49), respectively. Congruences (1.50)-(1.55) can be proved similarly.

Congruence (1.56) follows from (3.62) and (1.42). Congruence (1.57) follows from (3.63) and (1.43). Congruence (1.58) follows after replacing $n$ by $3 n+2$ in (3.62) and using (1.44). Congruence (1.59) follows after replacing $n$ by $3 n+1$ in (3.63) and using (1.45).

Remark 2. Although the first proof is much shorter, the second proof reveals more information. It establishes congruences modulo powers of 3 for the sequences $a_{i}(n)$ $(i \in\{3,4,5\})$, which may be useful for people who are interested in these sequences.

\section{REFERENCES}

[1] G.E. Andrews, Generalized Frobenius Partitions, Memoirs of the American Mathematical Society, No. 301, American Mathematical Society, Providence, RI, 1984.

[2] N.D. Baruah and B.K. Sarmah, Congruences for generalized Frobenius partitions with 4 colors, Discrete Math. 311 (2011), 1892-1902.

[3] N.D. Baruah and B.K. Sarmah, Generalized Frobenius partitions with 6 colors, Ramanujan J. 38 (2015), 361-382.

[4] J.M. Borwein, P.B. Borwein and F.G. Garvan, Some cubic modular identities of Ramanujan, Trans. Amer. Math. Soc. 343 (1) (1994), 35-47.

[5] H.H. Chan, L. Wang and Y. Yang, Congruences modulo 5 and 7 for 4-colored generalized Frobenius partitions, J. Aust. Math. Soc. 103 (2) (2017), 157-176.

[6] H.H. Chan, L. Wang and Y. Yang, Modular forms and $k$-colored generalized Frobenius partitions, to appear in Trans. Amer. Math. Soc. https://doi.org/10.1090/tran/7447

[7] D. Eichhorn and J.A. Sellers, Computational proofs of congruences for 2-colored Frobenius partitions, Int. J. Math. Math. Sci. 29 (2002), 333-340.

[8] F.G. Garvan and J.A. Sellers, Congruences for generalized Frobenius partitions with an arbitrarily large number of colors, Integers 14 (2014), Article A7.

[9] C. Gu, L. Wang and E.X.W. Xia, Congruences modulo powers of 3 for generalized Frobenius partitions with 6 colors, Acta Arith. 175 (2016), no. 3, 291-300.

[10] M.D. Hirschhorn, Some congruences for 6-colored generalized Frobenius partitions, Ramanujan J. 40(3) (2016), 463-471.

[11] M.D. Hirschhorn and J.A. Sellers, Infinitely many congruences modulo 5 for 4-colored Frobenius partitions, Ramanujan J. 40 (2016), 193-200.

[12] L.W. Kolitsch, An extension of a congruence by Andrews for generalized Frobenius partitions, J. Combin. Theory Ser. A 45 (1987), 31-39. 
[13] L.W. Kolitsch, A relationship between certain colored generalized Frobenius partitions and ordinary partitions, J. Number Theory 33 (1989), 220-223.

[14] L.W. Kolitsch, A congruence for generalized Frobenius partitions with 3-colors modulo powers of 3, in Analytic Number Theory: Proceedings of a Conference in Honor of Paul T. Bateman (Birkhäuser, Boston, MA, 1990), 343-348.

[15] L.W. Kolitsch, A different way to sort $M$-order, colored generalized Frobenius partitions, J. Number Theory 56 (1996), 188-193.

[16] B.L.S. Lin, New Ramanujan type congruence modulo 7 for 4-colored generalized Frobenius partitions, Int. J. Number Theory 10 (2014), 637-639.

[17] J. Lovejoy, Ramanujan-type congruences for three colored Frobenius partitions, J. Number Theory 85 (2000), 283-290.

[18] K. Ono, Congruences for Frobenius partitions, J. Number Theory 57 (1996), 170-180.

[19] P. Paule and C.S. Radu, The Andrews-Sellers family of partition congruences, Adv. Math. 230 (2012), 819-838.

[20] J.A. Sellers, Congruences involving generalized Frobenius partitions, Int. J. Math. Math. Sci. 16 (1993), 413-415.

[21] J.A. Sellers, New congruences for generalized Frobenius partitions with 2 or 3 colors, Discrete Math. 131 (1994), 367-374.

[22] J.A. Sellers, An unexpected congruence modulo 5 for 4-colored generalized Frobenius partitions, J. Indian Math. Soc., Special Volume to Commemorate the 125th Birth Anniversary of Srinivasa Ramanujan (2013), 97-103.

[23] L. Wang, Arithmetic identities and congruences for partition triples with 3-cores, Int. J. Number Theory 12 (2016), 995-1010.

[24] E.X.W. Xia, Proof of a conjecture of Baruah and Sarmah on generalized Frobenius partitions with 6 colors, J. Number Theory 147 (2015), 852-860.

[25] E.X.W. Xia, A Ramanujan-type congruence modulo 5 for 4-colored generalized Frobenius partitions, Ramanujan J. 39 (2016), 567-576.

[26] W.L. Zhang and C. Wang, An unexpected Ramanujan-type congruence modulo 7 for 4-colored generalized Frobenius partitions, Ramanujan J. 44(1) (2017), 125-131.

School of Mathematics and Statistics, Wuhan University, Wuhan 430072, Hubei, PeoPLE'S REPUBlic OF CHINA

E-mail address: wanglq@whu.edu.cn; mathlqwang@163.com 